\title{
SIMPLE BAYESIAN GENE NETWORK LEARNING IN POPULUS DROUGHT TRANSCRIPTOME DATA
}

\author{
Amir Almasi Zadeh Yaghuti ${ }^{\dagger}$, Ali Movahedi ${ }^{\dagger}$, Hui Wei, Weibo Sun, \\ Mohaddeseh Mousavi and Qiang Zhuge*
Co-Innovation Center for Sustainable Forestry in Southern China, Key Laboratory of Forest Genetics \& Biotechnology, Ministry of Education, College of Biology and the Environment, \\ Nanjing Forestry University, Nanjing 210037, China \\ Keywords: Gene network, Populus, Microarray data, NCBI
}

\begin{abstract}
Constructing a sensibly functional gene interaction network is highly appealing for better understanding system-level biological processes governing various Populus traits. Bayesian Network (BN) learning provides an elegant and compact statistical approach for modeling causal gene-gene relationships in microarray data. Therefore, it could come with the illumination of functional molecular playing in Biology Systems. In the present study, different forms of gene Bayesian networks were detected on Populus cellular transcriptome data. Markov blankets would likely be emerging at every possible gene regulatory Bayesian network level. Results showed that PtpAffx.1257.4.S1_a_at,1.0 hypothetical protein is the most important in its possible regulatory program. This paper illustrates that the gene network regulatory inference is possible to encapsulate within a single BN model. Therefore, such a BN model can serve as a promising training tool for Populus gene expression data for better future experimental scenarios.
\end{abstract}

\section{Introduction}

The development of the theory of causal modeling roots back to the 1950s, which has left some scientific community controversies. The core of these debates and controversies is the Markov condition/ assumption, an assumption made in the Bayesian probability theory. BN has been used to model chlordecone bioaccumulation in plants (Liber et al. 2020) to discover the best regulators of drought response (Lahiri et al. 2019) and to infer gene regulatory networks (Vignes et al. 2011). A Bayesian gene network consists of a digraph, which connecting regulatory genes to their targets, and elegantly encodes conditional independence between genes. Technically, BN is a combination of the Bayesian theorem with the directed acyclic graph (DAG). The DAG decomposes the joint probability distribution. Given the DAG, the joint probability distribution of the expression of 6 nodes/genes factorizes as follows:

$$
\mathrm{P}(\mathrm{A}, \mathrm{S}, \mathrm{E}, \mathrm{O}, \mathrm{R}, \mathrm{T})=\mathrm{P}(\mathrm{A}) \mathrm{P}(\mathrm{S}) \mathrm{P}(\mathrm{E} \mid \mathrm{A}, \mathrm{S}) \mathrm{P}(\mathrm{O} \mid \mathrm{E}) \mathrm{P}(\mathrm{R} \mid \mathrm{E}) \mathrm{P}(\mathrm{T} \mid \mathrm{O}, \mathrm{R})
$$

In general terms, this can be written as:

$$
p(x)=\prod_{i=1}^{n} p\left(x_{i} \mid \text { parents }\left(x_{i}\right)\right) \text {. }
$$

In a $\mathrm{BN}$, every gene must have a conditional probability table (CPT). For each gene, CPT indicates all the possible combinations of values of the parent genes. Each possible combination is called an Instantiation of the parent set. A BN structure is usually obtained from the data using score-based or constraint-based approaches (Koller and Friedman 2009). Score-based algorithms maximize the BN likelihood, using Markov chain Monte Carlo (MCMC); to search the space of network structures, it operates on edge additions, deletions, or inversions. Score-based algorithms

\footnotetext{
*Author for correspondence: <qzhuge@ njfu.edu.cn>.
} 
have been reported to fit well on simulated genetics and genomics data (Tasaki et al. 2015). However, the present researchers initially learned the network's undirected skeleton using repeated conditional independence tests in constraint-based approaches. Then by resolving directional constraints, each edge direction was assigned (v-structures and acyclicity) to the skeleton. Genetic mapping of multiple complex traits has been done (Scutari et al. 2014). Due to its complexity, Bayesian gene network inference is feasible for systems of at most a few hundred genes or variables through conventional algorithms (Beckmann et al. 2018, Wang et al. 2019). The topology of the network of BN encodes conditional independence assertions. Therefore, it was possible logically find a set of possible gene regulators out of entire gene expression data. In the present study, it was tried to find those genes in the Populus genome.

Drought is pivotal abiotic stress that affects plant development and poplar productivity (Hamanishi et al. 2015). Therefore, poplar breeding requires an understanding of the underpinning molecular regulatory machines controlling poplar resistance to drought stress. Molecular studies have revealed that drought stress might take place in different plant tissues. For instance, many plant transcriptomic studies have addressed transcriptional changes in roots (Cohen et al. 2010, Lorenz et al. 2011, Stolf-Moreira et al. 2011, Dash et al. 2018) due to drought stress over different genotypes (Cohen et al. 2010, Stolf-Moreira et al. 2011, Cao et al. 2014, Hamanishi et al. 2015, Jia et al. 2016). Moreover, gene network analysis on poplar root transcriptome revealed a hierarchical-like gene network structure in which the highest hierarchical level 2,934 genes, affected by nine super hubs (super genes) (Hamanishi et al. 2015) following different levels of drought stress (Cohen et al. 2010, Stolf-Moreira et al. 2011).

Here, microarray-based transcriptomic Bayesian gene network analysis of drought-resistant in black poplar genotypes measured in well-watered, moderate drought, severe drought, and postdrought re-watering conditions has been reported. Understanding the characteristics of poplar gene regulation of drought resistance, including various molecular interaction processes, elaborate functional knowledge of genes underlying this stress-induced phenomenon would be conducted.

\section{Materials and Methods}

Using the GEOquery package (Davis and Meltzer 2007), information directly from the GEO database with accession number GSE76322 was downloaded in this research. Initially, data dimensions were $61413 \times 18$. To run the Bayesian regulatory network, the probes with the highest variances were selected. Finally, the data dimension was reduced to $2210 \times 18$ (Table 1). The bnlearn package was used to infer the regulatory Bayesian network on the data. To determine the best network structure, the hill-climbing algorithm was used. The result of bnlearn was considered as a regulatory Bayesian network. The number of nodes, edges, Markov blanket (MB) size, neighborhood size, and the learned network's branching factor were calculated in this study. It has been assumed that with the broader MB size, the giant module network could be detected. In addition, with the bnlearn package help, the adjacency matrix with Cytoscape-based aMatReader software was imported to Cytoscape. Using the Network Analyzer plugin, a comprehensive set of topological parameters such as number of nodes, edges, network diameter, radius and clustering coefficient, neighborhood connection, shortest path length, number of familiar neighbors, the degree distribution, the centripetal proximity parameter, etc. were calculated for the present network. 
Table 1. Estimation of structural Bayesian network parameters with Hill Climbing algorithm.

\begin{tabular}{ll}
\hline Parameters & values \\
\hline No. of nodes & 2210 \\
No. of arcs (Edges) & 1000 \\
Undirected edges & 0 \\
Directed arcs & 1000 \\
MB & 0.92 \\
NS & 0.90 \\
BF & 0.45 \\
Penalization coefficient & 1.445186 \\
No. of tests & 4647736 \\
\hline MB: Markov Blanket, NS: Neighborhood Size, BF: Branching Factor, HC: Hill Climbing.
\end{tabular}

\section{Results and Discussion}

In general, for high-dimensional genetics and genomics data, BN learning is challenging since the number of expected networks scales up exponentially with the number of genes. The computational cost of conventional $\mathrm{BN}$ inference could be a prohibitive burden (Wang et al. 2019). Table 1 represents the general parameters of the observed probe-based Bayesian regulatory network. The number of probes (nodes), as can be seen in Table 1, is 2210, which is higher than the number of directed edges (1000) that is logical in this context. This would indicate that some orphan probes in the network or some nodes are associated averagely high with other nodes. One of the main parameters of detected $\mathrm{BN}$ is the $\mathrm{MB}(0.45)$. It can be declared that $\mathrm{MB}$ essentially and explicitly is a minimum set of genes that would block all back-door paths that could be discovered from learned BN. The MB of a gene includes its parents, children, and co-parents. Parents in the MB separate the impact of specific disturbance on the outcome from the rest of the gene network. Therefore, this concept can be used to select a smaller set of relevant genes in highdimensional problems. The MB is proven to be highly effective for feature reduction in highdimensional problems, sometimes reducing the number of variables a thousandfold without any loss of accuracy (Aliferis et al. 2003, Fu and Desmarais 2010, Shen et al. 2008, Tan and Liu 2013). It has been shown that the MB establishment is also instrumental in creating gene module networks. In other words, one could say that number of MBs and their sizes in BN may reflect the sense of modularity in the network. Parents of genes in the BN are the connections that would reflect causalities in the gene network. By identifying the MBs in different sizes over all genes in the gene network, one can quickly draw different gene sizes that cohesively function together, e.g., module network.

Figure 1 illustrates seven genes in a postulated gene regulatory BN. These seven genes constitute a structure which is called MB. The U1, Um genes are parents of the X gene, and Y1 and Yn genes are Childs of the X gene in which the other two parents, e.g., Z1j and Znj genes, share paternity with the $\mathrm{X}$ gene. This structure demonstrates some cohesive acting of genes, which will be called them up as gene modules. Finding these structures in BN would be biologically appealing. In the present study, it was turned out that many MB are cohesively overlapped. Therefore, a general picture of gene modules was not seen. It is expected that some grape-like structures, i.e., gene modules due to $\mathrm{MB}$; such structures were not visually turned up on the learned BN. The main finding of this study, e.g., PtpAffx.1257.4.S1_a_at,1.0, hardly bordered as a 
specific set of probes as gene module. In other words, this probe (and its belonging gene) might be involved in many other MB as well. This may indicate the level of complication of doughtiness on the poplar genome. In other words, drought stress has caused many different biological pathways actions. In every gene regulatory BN network, the total connectivity for each gene/probe could be found, and this measure in BN can be split up into out-degree and in-degree. Fig. 2, Supplementary Tables 1 and 2 address some topological aspects of learned BN. Putting this in a biological context, it could be seen that nodulizing BN has been hardly achieved. One reason could be the fact that drought stress affected many different functional parts of the poplar genome.

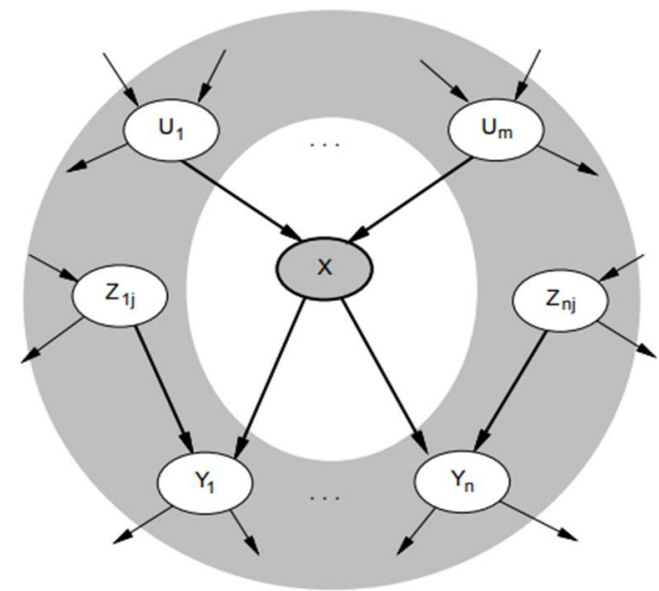

Fig. 1. MB of a given postulated gene $\mathrm{BN}$ showing $\mathrm{MB}$ of a node/probe/gene and the set containing the node/gene's parents, children, and co-parents.

However, the distribution of gene out-degree has been much higher than gene in-degree. This is biologically supported as out-degree probes/genes could be considered as a regulator, which patently indicates that a small number of genes had many out-degrees. Some of these high-outdegree genes are shown in Supplementary Table 2. The results of this table can be used for further analysis, for example, utilizing suitable gene set enrichment analysis. A general feature of many gene networks is their nature of scale-free topologies addressing a gene degree distribution that can be shown with a function of power-law, e.g., $P(\mathrm{k})=C k^{-\alpha}$, in which $\mathrm{P}(\mathrm{k})$ is the randomly selected gene having degree $\mathrm{k}$ (or $\mathrm{k}$ connections), $\alpha$ is the power-law exponent, and the constant $\mathrm{C}$ is a Riemann's zeta function, e.g., $\sum_{n=1}^{\infty} \frac{1}{n^{s}}$ (s = complex variable and $\mathrm{n}=$ integer) normalizing the power law probability distribution, e.g., $\sum_{k=1}^{\infty} P(k)=1$. In scale-free gene network topologies, most genes generally have relatively few interactions reflected as a lower degree. In contrast, a small number of genes, so-called 'hubs' genes, have a higher degree. This may prohibit seeing clean-up gene module in studies similar to the pr4esent study.

To predict gene function in poplar gene expression data using DNA microarray datasets, private or publicly available databases are needed. The present method allows users to extract genes involved in biological processes, which could be valuable for understanding similar tree species mechanisms (Ogata et al. 2009). The mode of genes in terms of being regulator or regulated is not reflected in this type of database and could make the practical biological application of such databases restrictive. The public domain data have been used in the present study and checked up on a new hypothesis in the Populus genome. This data are used to create 
Supplementary Table 1. Simple parameters in network Analyzer.

\begin{tabular}{ll}
\hline Clustering coefficient & 0.000 \\
\hline Number of nodes & 2211 \\
Connected components & 1 \\
Network diameter & 1 \\
Network radius & 1 \\
Shortest paths & $2210(0 \%)$ \\
Characteristics path length & 1 \\
The average number of neighbors & 1.999 \\
Network density & 0.0 \\
Isolated nodes & 426 \\
number of self-loops & 0 \\
multi-edge node pairs & 0 \\
Analysis times (Sec) & 0.874 \\
\hline
\end{tabular}

Supplementary Table 2. The CytoHubba plugin chose the first 30 genes in the MCC method.

\begin{tabular}{ll}
\hline PtpAffx.1257.4.S1_a_at,1.0 & Hypothetical protein /// hypothetical protein \\
PtpAffx.1258.1.S1_s_at,1.0 & Hypothetical protein \\
PtpAffx.1258.4.S1_s_at,1.0 & Aktin9 \\
PtpAffx.1259.1.S1_s_at,1.0 & Hypothetical protein \\
PtpAffx.12595.1.A1_s_at,1.0 & Hypothetical protein \\
PtpAffx.126167.1.S1_at,1.0 & Branched-chain amino acid aminotransferase-like protein \\
PtpAffx.126235.1.S1_at,1.0_ & Auxin-responsive family protein \\
PtpAffx.12628.1.S1_at,1.0 & Metal handling. Binding, chelation, and storage \\
PtpAffx.126599.1.A1_s_at,1.0 & Arginine decarboxylase \\
PtpAffx.1263.1.A1_a_at,1.0 & Hypothetical protein \\
PtpAffx.12675.3.A1_s_at,1.0 & Unknown protein; predicted by gene finder \\
PtpAffx.12675.3.A1_a_at,1.0 & Hypothetical protein \\
PtpAffx.12684.1.S1_at,1.0 & pyrophosphate-dependent 6-phosphofructose-1-kinase, putative \\
PtpAffx.12676.1.S1_at,1.0 & MAC/Perforin domain-containing protein \\
PtpAffx.1269.1.A1_x_at,1.0 & Putative thioredoxin \\
PtpAffx.1269.1.A1_a_at,1.0 & Putative thioredoxin \\
PtpAffx.12712.1.S1_at,1.0 & Hypothetical protein \\
PtpAffx.127056.1.A1_at,1.0 & Signal transducer activity \\
PtpAffx.12745.1.A1_at,1.0 & Unknown protein \\
PtpAffx.1273.1.S1_at,1.0 & Copper ion binding, laccase activity, \\
PtpAffx.127847.1.A1_at,1.0 & DNA binding protein \\
PtpAffx.127644.1.A1_s_at,1.0 & Hypothetical protein \\
PtpAffx.12800.1.S1_a_at,1.0 & Hypothetical protein \\
PtpAffx.127878.1.A1_at,1.0 & Unknown protein \\
PtpAffx.1286.2.S1_s_at,1.0 & S-adenosylmethionine decarboxylase \\
PtpAffx.1286.1.S1_s_at,1.0 & S-adenosylmethionine decarboxylase, putative \\
PtpAffx.12874.1.S1_at,1.0 & Hypothetical protein \\
PtpAffx.1286.5.S1_s_at,1.0 & Hypothetical protein \\
PtpAffx.128895.1.S1_at,1.0 & Lysine-ketoglutarate reductase/saccharopine dehydrogenase \\
\hline
\end{tabular}


This may prohibit seeing clean-cut gene modules in studies similar to our study.

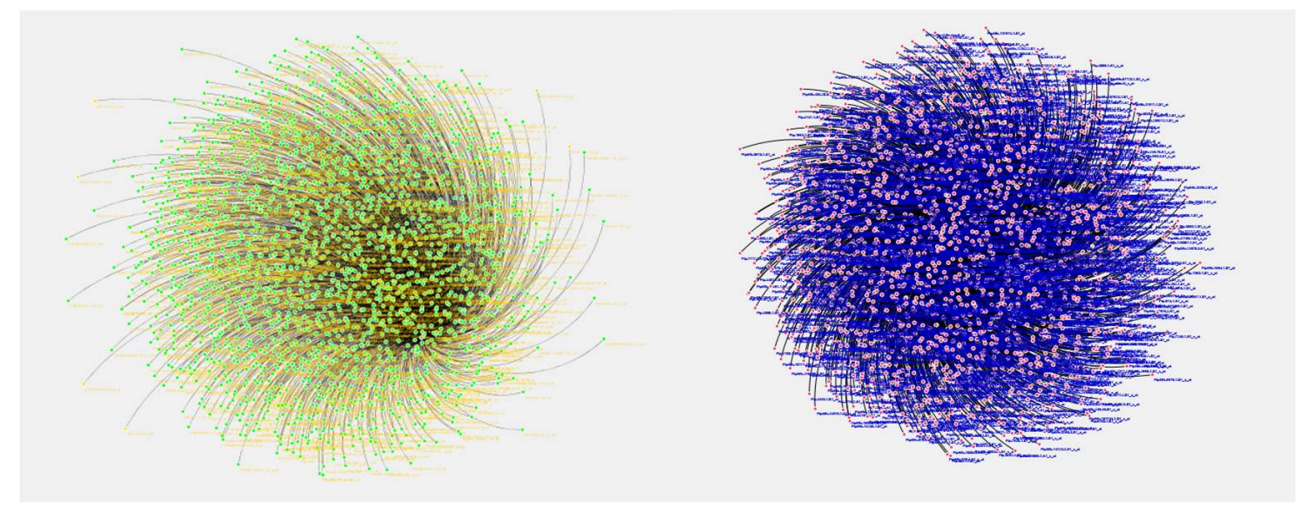

Fig. 2. The snapshot of gene connectivity in learned BN in two graph forms.

a gene network (Cai et al. 2014) who assigned the functional category modules of Populus cell wall genes using a combination of a genome-wide Populus gene co-expression network (PGCN) and module detection and gene ontology (GO) enrichment analysis. However, this study revealed a probabilistic one instead of a Pearson-based gene correlation matrix. The data did not find any topological properties of the network indicating scale-free and modular behavior. As it turned out, the researchers intensely relied on $\mathrm{BN}$ topological properties, e.g., $\mathrm{MB}$, to address possible modules in the present data. Figures 1 and 2 showed no sign of modules.

In the domain of gene expression high throughput data, probabilistic dependencies arise because genes are related in different ways (e.g., through common phenotype, logical connections, pathway connections, through (non-causal) physical laws, constrained of thermodynamic laws or structural boundary conditions). This may prohibit seeing clean-cut gene modules in studies like the present study. Figure 1 demonstrates that no sign of modules is trackable to pick one out of the gene regulatory network. The method used in this study could likely help to identify and characterize cell wall-related genes in Populus. Zhang and Yin (2016) applied graph-based theory and identified 14 probe-sets/genes related to B plasmodesmata GO cellular component term and many other genes for other tissues. However, the graph-based networks of the different tissues have shown different topological properties. They illustrate that genes in the root network were the most highly co-expressed. Whereas the leaf genes were the weakest co-expressed, and those in wood were in the intermediate. These topological network differences provide some unseen epigenetic mechanisms in different tissues. The genetic mechanism underlying the different topological properties among these tissues remains unknown and is worthy of further investigation in future studies. In general, finding gene modules in Populus data has an almost long history (Gronlund et al. 2009, Liu et al. 2016, Han et al. 2020). In most of them, the co-expression analyses have been the cornerstone in finding modules of co-expressed genes; gene modules display distinct expression profiles. However, addressing hub genes in this kind of study is a difficult task. For example, Fig. 2 shows how genes are cohesively related, and therefore, it is pretty challenging to pick up and address some genes which actively responded to drought.

In the MCC algorithm, it is possible to highlight motivated biological molecules in the network. Some yellow circle nodes in figure 3 display the top ten most connected hub genes. Figure 3 reveals a close relationship between other genes and these top 10 genes, making it challenging to report some general gene module structures in achieved data. However, in this 
study, the applied BN model should be regarded as a conjecture, which should be tested using more and better posed high dimensional data.

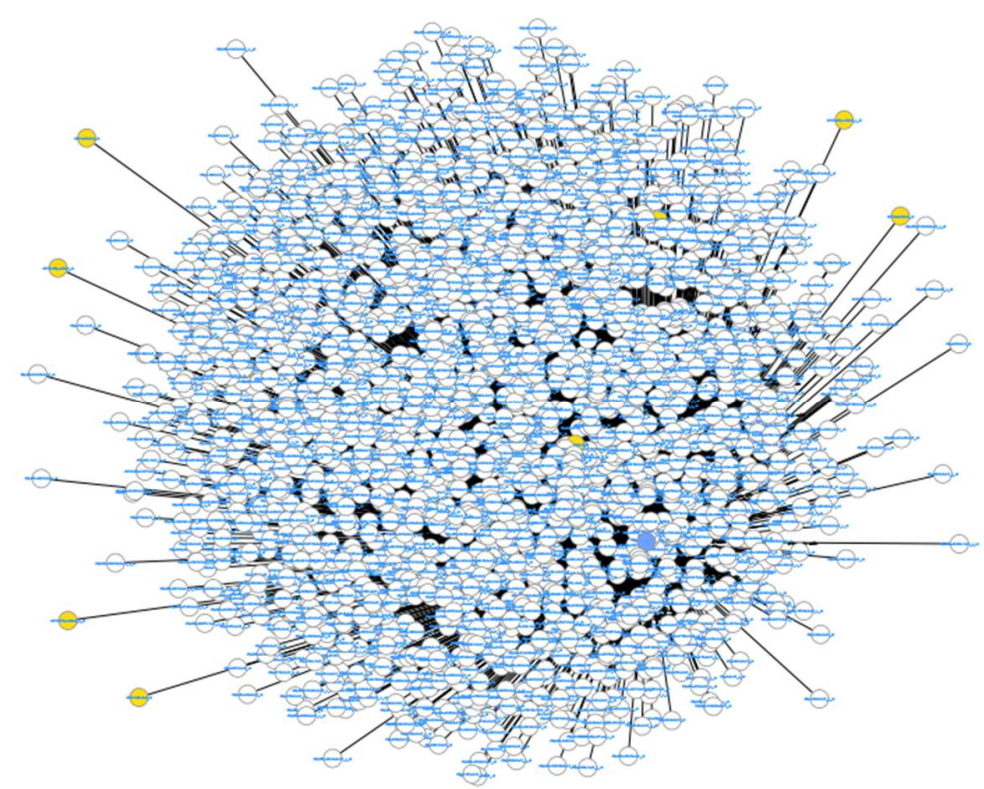

Fig. 3. Highlighting top 10 genes with their neighbors using MCC algorithm.

Poplar has emerged as an ideal model system for studying woody plants. For better understanding the biological processes underlying various poplar traits, e.g., wood development, a comprehensive functional gene interaction network is highly needed. To more effectively screening down, Some highly connected genes have been used in this study. Poplar Gene offers comprehensive functional interactions and extensive poplar gene functional annotations. Seminal research on the poplar genome shows that a relatively small genome, quick growth trend, and simple genome clonal manipulation are the interesting biological features that have made poplar a long-lived forest tree model system (Liu et al. 2016). Drought stress is the leading cause of plant loss worldwide, and drought is an essential environmental factor affecting almost all plant species (Dash et al. 2018). As a model, Populus provides an opportunity to study the stress response in a perennial tree growing as a commercial biomass product to produce carbon-neutral energy.

In the present study, as can be seen in Supplementary Table 2, many genes turned out to be highly connected ones: hub genes. Moreover, their annotations reveal that they do not have comprehensive biological support (as indicated by hypothetic terms). Therefore, it was not easy to see their possible protein interaction in the STRING database. Protein-protein interaction networks play an essential role in understanding the system level of cellular processes. These networks can filter and evaluate functional genomic data and create an intuitive platform for annotating proteins' structural, functional, and evolutionary properties. The aktin three genes were fed to the STRING database, and the grid of protein interaction was extracted. STRING is a protein-protein interactions database (the known and predicted one) and a retrieval search tool. The results of drawing interactive networks in the STRING database are shown in Fig. 4. The result of the protein network was architecturally different from those obtained from the Bayesian gene network. In other words, some inconsistency and discrepancy in the results of the network learned 
by STRING were observed compared to the learned BN network, which shows that posttranscriptional modification may play crucial roles in regulating gene expression in the Populus genome.

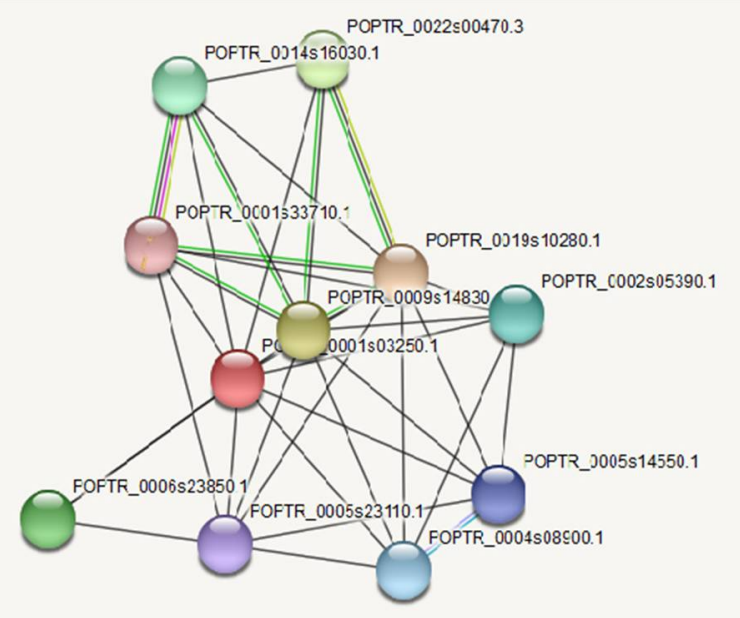

Fig. 4. Network of protein-protein interactions by STRING database information.

This study logically relied on Populus mRNA data; therefore, talking about the PPI network does not make much sense. However, it is believed that the whole body or tissue-specific proteinprotein interactome (PPI) map could highlight drought tolerance in the Populus. Large-scale studies of PPIs accompanying the species-specific database support the interplay of cascading translational protein levels for drought-related stress adaptive mechanisms in Populus. Interestingly, many genes that became hub genes in this study were biologically involved in photosynthesis-related processes and light reactions. These are the key findings of this study. Most likely abiotic processes like drought act on the biological system level of Populus. I

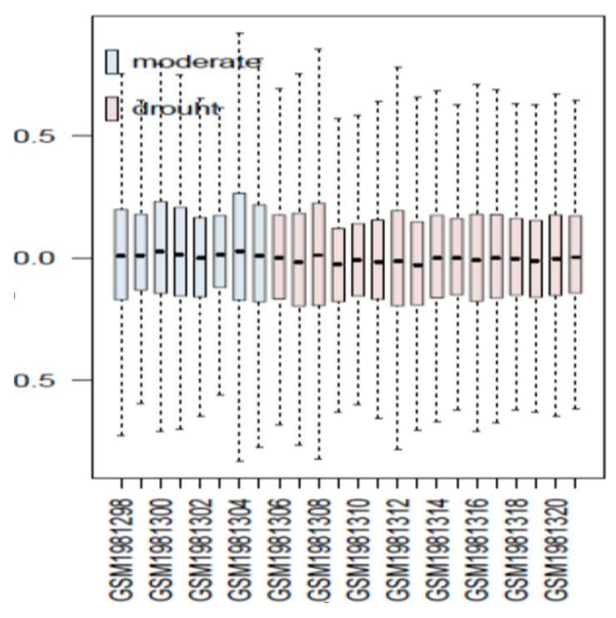

Fig. 5. Boxplot of GSE764322 using in this study. 
Boxplot is a visualization tool of microarray data. Each box stretches from the lower hinge defined as the 25th percentile to the upper hinge - the 75th percentile- and the median is delineated as a line across the box. This figure has appeared were taken in microarray experiment data at the top of other figures. However, some so-called downside-up approaches were taken in a way that is shown at the bottom of the table to make it visually much more transparent than the treatments used in applied GSE76322, which did not impart much more diversity on the gene expression (Fig. 5). However, this sort of view should not mislead researchers, as in this study, many probes turned out to be differently expressed. It is believed that BN can decipher information on Populus microarray data, which should be figured out in subsequent studies.

For the first time, a BN was learned out on Populus DNA microarray data. This study identified a gene network in the Populus to highlight candidate genes used as regulator genes. The top 30 hub genes, in which some of them had low if any, valid biological information were identified. It was noted that the MB in BN of gene regulation could be assumed to narrow down the whole gene network complexity. Statistically speaking, the existence of an MB means external genes are conditionally independent of internal genes and vice versa. This is biologically appealing. Despite extensive physiological and morphological descriptions of the Populus response to drought, little work has been done to explain the differences in gene levels and examine the similarity of the stress response between this perennial and the annual crop. Because poplar genome sequences and poplar microarrays are now available, a bridge can be made between quantitative trait locus mapping approaches, the candidate gene approach, and transcription.

\section{Acknowledgments}

This study was supported by the National Science Foundation of China (No. 31570650) and the Priority Academic Program Development of Jiangsu Higher Education Institutions.

\section{References}

Aliferis, Constantin F, Tsamardinos, Ioannis, Statnikov, Alexander (Eds.) 2003. HITON: a novel Markov Blanket algorithm for optimal variable selection. AMIA annual symposium proceedings. p. 21

Beckmann ND, Lin W-J, Wang M, Cohain AT, Wang P, Ma W, et al. 2018. Multiscale causal network models of Alzheimer's disease identify VGF as a key regulator of disease. bioRxiv: p. 458430.

Cai B, Li CH and Huang J 2014. Systematic identification of cell-wall related genes in Populus based on analysis of functional modules in co-expression network. PLoS One 9(4): e95176. DOI: 10.1371/ journal.pone.0095176.

Cao X, Jia J, Zhang C, Li H, Liu T, Jiang X et al. 2014. Anatomical, physiological and transcriptional responses of two contrasting poplar genotypes to drought and re-watering. Physiologia plantarum 151(4): 480-494. DOI: $10.1111 /$ ppl.12138.

Cohen D, Bogeat-Triboulot MB,Tisserant E, Balzergue S, Martin-Magniette ML, Lelandais G et al. 2010. Comparative transcriptomics of drought responses in Populus: a meta-analysis of genome-wide expression profiling in mature leaves and root apices across two genotypes. BMC genomics 11(1): p. 630. DOI: 10.1186/1471-2164-11-630.

Dash M,Yordanov YS, Georgieva T, Wei H and Busov V 2018. Gene network analysis of poplar root transcriptome in response to drought stress identifies a PtaJAZ3PtaRAP2.6-centered hierarchical network. PLoS One 13(12): e0208560. DOI: 10.1371/journal.pone.0208560.

Davis S and Meltzer PS 2007. GEOquery: a bridge between the Gene Expression Omnibus (GEO) and Bioconductor. Bioinformatics 23(14): 1846-1847. 
Fu, Shunkai, Desmarais, Michel C. (Eds.) 2010. Markov blanket based feature selection: a review of past decade. Proceedings of the world congress on engineering, 2010: Newswood Ltd (1).

Gronlund A, Bhalerao RP and Karlsson J 2009. Modular gene expression in poplar: a multilayer network approach. New Phytol. 181(2): 315-322. DOI: 10.1111/j.1469-8137.2008.02668.x.

Hamanishi ET, Barchet GL, Dauwe R, Mansfield SD and Campbell MM 2015. Poplar trees reconfigure the transcriptome and metabolome in response to drought in a genotype- and time-of-day-dependent manner. BMC genomics 16(1): p. 329. DOI: 10.1186/s12864-015-1535-z.

Han X, An Y, Zhou Y, Liu C, Yin W and Xia X 2020. Comparative transcriptome analyses define genes and gene modules differing between two Populus genotypes with contrasting stem growth rates. Biotechnology for Biofuels 13(1): 1-21.

Jia J, Li S, Cao X, Li H, Shi W, Polle A et al. 2016. Physiological and transcriptional regulation in poplar roots and leaves during acclimation to high temperature and drought. Physiologia Plantarum 157(1): 3853. DOI: 10.1111/ppl.12400.

Koller and Friedman 2009. Probabilistic Graphical Models - Principles and Techniques.

Lahiri A,Venkatasubramani PS and Datta A 2019. Bayesian modeling of plant drought resistance pathway. BMC Plant Biol. 19(1): p. 96. DOI: 10.1186/s12870-019-1684-3.

Liber Y, Cornet D,Tournebize R, Feidt C, Mahieu M, Laurent F and Bedell JP 2020. A Bayesian network approach for the identification of relationships between drivers of chlordecone bioaccumulation in plants. Environ. Sci. Pollut. R. 27(33): 41046-41051. DOI: 10.1007/s11356-019-07449-0.

Liu Q,Ding C, Chu Y, Chen J, Zhang W, Zhang B, et al. 2016. PoplarGene: poplar gene network and resource for mining functional information for genes from woody plants. Sci. Rep. 6: p. 31356. DOI: 10.1038/srep31356.

Lorenz WW, Alba R,Yu YS, Bordeaux JM, Simoes M and Dean JF 2011. Microarray analysis and scale-free gene networks identify candidate regulators in drought-stressed roots of loblolly pine $(P$. taeda L.). BMC genomics 12: p. 264. DOI: 10.1186/1471-2164-12-264.

Ogata Y,Suzuki H and Shibata D 2009. A database for poplar gene co-expression analysis for systematic understanding of biological processes, including stress responses. J. Wood Sci. 55(6): 395-400. DOI: 10.1007/s10086-009-1058-9.

Scutari M, Howell P, Balding DJ, and Mackay I 2014. Multiple Quantitative Trait Analysis Using Bayesian Networks. Genetics 198(1): 129-137. DOI: 10.1534/genetics.114.165704.

Shen, Jianqiang; Li, Lida; Wong, Weng-Keen (Eds.) 2008. Markov Blanket Feature Selection for Support Vector Machines. AAAI, 2008 (8).

Stolf-Moreira R,Lemos EGM,Carareto-Alves L,Marcondes J,Pereira SS,Rolla AAP et al. 2011. Transcriptional Profiles of Roots of Different Soybean Genotypes Subjected to Drought Stress. Plant Mol. Biol. Rep. 29(1): 19-34. DOI: 10.1007/s11105-010-0203-3.

Tan, Yuan; Liu, Zhifa (Eds.) 2013. Feature selection and prediction with a Markov blanket structure learning algorithm. BMC bioinformatics, 2013: Springer (14).

Tasaki S, Sauerwine B, Hoff B, Toyoshiba H and Gaiteri C and Chaibub Neto E 2015. Bayesian Network Reconstruction Using Systems Genetics Data: Comparison of MCMC Methods. Genetics 199(4): 973U128. DOI: 10.1534/genetics.114.172619.

Vignes M,Vandel J,Allouche D,Ramadan-Alban N,Cierco-Ayrolles C, Schiex T et al. 2011. Gene Regulatory Network Reconstruction Using Bayesian Networks, the Dantzig Selector, the Lasso, and Their MetaAnalysis. Plos One 6(12): e29165. DOI: 10.1371/journal.pone.0029165.

Wang LF,Audenaert P and Michoel T 2019. High-Dimensional Bayesian Network Inference From Systems Genetics Data Using Genetic Node Ordering. Front Genet 10: p. 1196. DOI: 10.3389/fgene.2019.01196.

Zhang HP and Yin TM 2016. Identifying candidate genes for wood formation in poplar based on microarray network analysis and graph theory. Tree Genet Geno. 12(3): p. 61. DOI: 10.1007/s11295-016-1016-9. 